\title{
Concept and design of a UAS-based platform for measurements of RF signal-in-space
}

\author{
Thorsten Schrader ${ }^{1}$, Jochen Bredemeyer ${ }^{2}$, Marius Mihalachi ${ }^{1}$, Jan Rohde ${ }^{1}$, and Thomas Kleine-Ostmann ${ }^{1}$ \\ ${ }^{1}$ Physikalisch-Technische Bundesanstalt (PTB), Braunschweig, Germany \\ ${ }^{2}$ FCS Flight Calibration Services GmbH, Braunschweig, Germany
}

Correspondence to: Thorsten Schrader (thorsten.schrader@ptb.de)

Received: 15 January 2016 - Revised: 26 April 2016 - Accepted: 17 May 2016 - Published: 28 September 2016

\begin{abstract}
Field strength or signal-in-space (SIS) measurements have been performed by using manned helicopters, aircrafts or from ground level using extendable masts. With the availability of unmanned aerial systems (UAS) such as multicopters a new versatile platform for SIS measurements is deployable. Larger types show up to eight individually driven electric motors and controllers (therefore called octocopter). They provide the ability to fly along predefined traces, to hover at waypoints and to initiate other actions when those have been reached. They provide self-levelling and stabilisation and moreover, they may gear at a point of interest regardless of their actual position, e.g. during their flight around a tower. Their payload mainly depends on the platform size and allows integration of complex measurement equipment. Upgrading their navigation capabilities including state-ofthe-art global navigation satellite system (GNSS) and ground station transmitter (real-time kinematic - RTK) enables precise localisation of the UAS. For operation in electromagnetic harsh environments a shielding can be considered and integrated into the concept.

This paper describes concept and design of an octocopter and its instrumentation, along with applications in recent projects, in which we measure and validate terrestrial navigation systems applied in air traffic and the weather forecast services. Among those are instrumentation landing systems (ILS), VHF omnidirectional radio ranges (VOR), airport traffic and weather radars as well as military surveillance radars, and UHF wind profilers. Especially to investigate the possible interaction of VORs and radars with single wind turbines (WT) or wind power plants has become a major request of economy, military and politics. Here, UAS can be deployed to deliver measurement data investigating this interaction. Once developed and setup to a certain extent, UAS are easy
\end{abstract}

and cost-efficient to operate. Nonetheless, due to their compact size, UAS will have rather low interaction with the electromagnetic field to be measured compared to the operation of manned helicopters.

\section{Introduction}

Field strength or signal-in-space (SIS) measurements have been performed by using manned helicopters, aircrafts or with extendable masts on ground level. Also helium-filled balloons and blimps have been used in the past. Drawbacks of their operation are high costs, fast movement (no repetitive measurement samples can be taken at the same spot), limited maneuverability, long setup time, or, in case of a mast, limited air space to be covered. Yet, with the availability of unmanned aerial systems (UAS) such as multicopters a versatile platform can be deployed. They bring along several features that drastically improve SIS measurements. Possible fields of application may include (but are not limited to) aerial photography, infrared spectroscopy and thermometry, surveillance, inspection and service, surveying, etc. Here, the UAS is used for precision electromagnetic field measurements in a broad frequency range. This includes measurement of absolute electromagnetic field levels and field distributions, on-site measurement of antenna patterns, measurement of communication channels and networks as well as their interference with wind turbine installations. Currently, we are conducting two projects for RF field strength and interaction measurements. The first is performed together with the German Federal Supervisory Office for Air Traffic Control (BAF) in Langen, Germany. In that project, mainly the absolute field strength level of instrument landing sys- 
tems (ILS) is of interest (primary the localiser part), which can not be assessed sufficiently by conventional flight inspection (FI). In the second project (WERAN project, German abbreviation for "Measuring the potential interaction of wind turbines with terrestrial navigation and radar systems", Schrader et al., 2015), supported by the Federal Ministry of Economic Affairs and Energy on the basis of a decision by the German Bundestag (grant: 0325644A), the potential interaction between wind turbines and terrestrial navigation systems and radar used in civil and military air traffic control, weather radar and weather forecast wind profiler is investigated. Therefore, the German Air Traffic and Navigation Service Provider (Deutsche Flugsicherung DFS GmbH) and the German Weather Service (Deutscher Wetterdienst, DWD) are unfunded partners in the WERAN project. Together with funded partners, the Institute for Foundations of Electrical Engineering and Measurement Technology, Leibniz University of Hanover, Germany, and steep $\mathrm{GmbH}$, Bonn, Germany, we conduct the WERAN project.

Rather than developing the octocopter from scratch, a commercially available platform "ARF-OktoXL" from HiSystems GmbH, Moormerland, Germany (http://www. mikrokopter.de), has been chosen and customised according to our needs (Fig. 1).

The manufacturer also provides a convenient GUI (cp. Fig. 2) to setup the UAS (check motor controls etc.) and the measurement campaign including the GNSS based flight track, way points, points of interest, etc. (see Sect. 2 for more information).

Our main focus is to make latest multicopter technology available for the precision measurement of electromagnetic fields.

When starting the UAS research program "ARCASS" (advanced remote-controlled airborne sensor system) in our department of High Frequency and Fields, we already had experience in the operation of remotely controlled model helicopters of bigger size ( $1.6 \mathrm{~m}$ rotor diameter). Seen from the retrospective, our experiences with helicopters helped to some extent to safely operate the octocopter during the experimental setup. It has to be noted here that pilots shall apply for an official permit issued by the Federal State Authority for Traffic to operate any sort of UAS, regardless of their commercial or scientific usage. The flight skills of the pilot are assessed by the local authorities. Moreover, the owner of the ground has to give his agreement for start and landing on his premises. Other official rules may apply for flight paths, maximum heights, areas etc. Local regulations must be obeyed.

In Sect. 2 we describe the design principles of the flying platform. Both the RF instrumentation and the improved GNSS receiver on-board are given in Sect. 3. In Sect. 4 we present first measurement results as prof-of-concept.

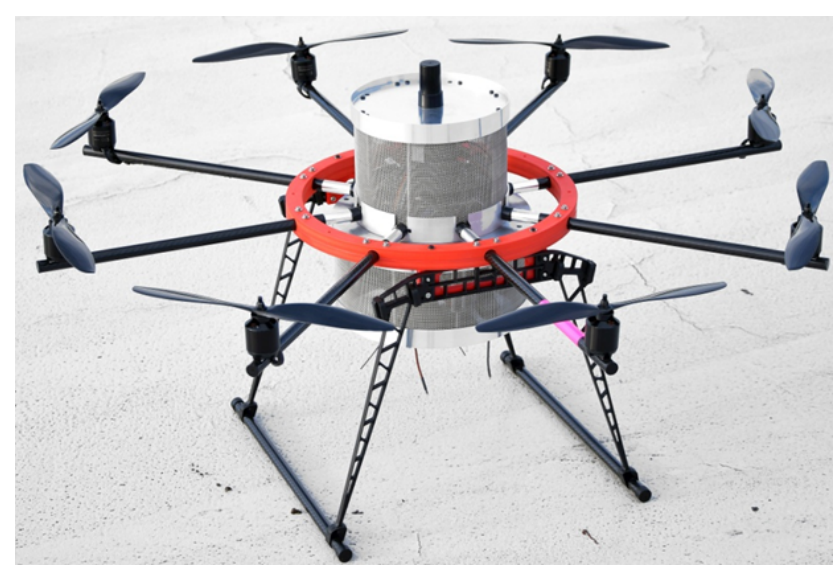

Figure 1. Photograph of electromagnetically shielded octocopter.

\section{Design of flying platform}

ARCASS always uses a standard flying platform (UAS) with advanced precision localisation (Mihalachi et al., 2015). However, dependent on the task, the instrumentation, namely the sensor head, is exchanged. The sensor head consists of receiving antenna and RF frontend with up to three coherent channels. Each sensor head is specifically designed for the desired frequency band and task. The sensor system consists of multiple sensor heads that can be operated consecutively one after another (but not in parallel) whereas the data sampling and recording unit is kept unchanged at any time.

A quick-lock system is used, on the one hand ensuring a fast exchange of the different heads, and on the other, to provide a consistent orientation of the sensors with respect to the UAS. This is a primary condition to be fulfilled in order to assign calibration factors to the whole setup consisting of sensor head and UAS. A second condition is based on the ability of the UAS, to always "face" a predefined point of interest. As an example, we discuss the measurement of an antenna pattern of a broadcast tower. When the UAS is orbiting the antenna mast at constant height, the UAS simultaneously rotates around its vertical axis with regard to the actual position instead of maintaining a constant yaw angle. By this procedure the receiving antenna pattern of the UAS remains almost constant, of course with some uncertainty.

\subsection{Mechanical setup and EMC considerations}

Besides the possibility to calibrate the receiving antenna factor and other electrical requirements such as shielding the setup and the design of our octocopter "PTBee" (cp. Figs. 1, 3 ) has to meet additional practical requirements. These include smooth operation, a quick exchange of batteries and general fulfillment of Electromagnetic Compatibility (EMC). On the one hand, motor controller switching, high motor line and battery currents could cause internal EMC problems such as distortions of the magnetic sensor to control 


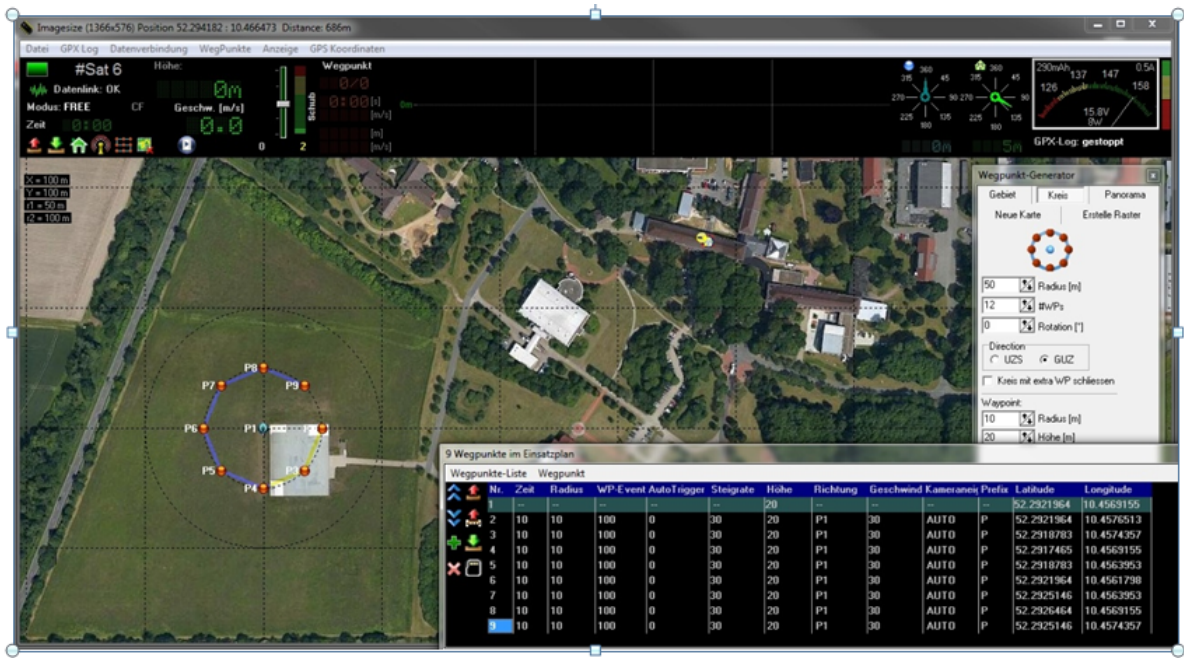

Figure 2. Screenshot of GUI for campaign planning.

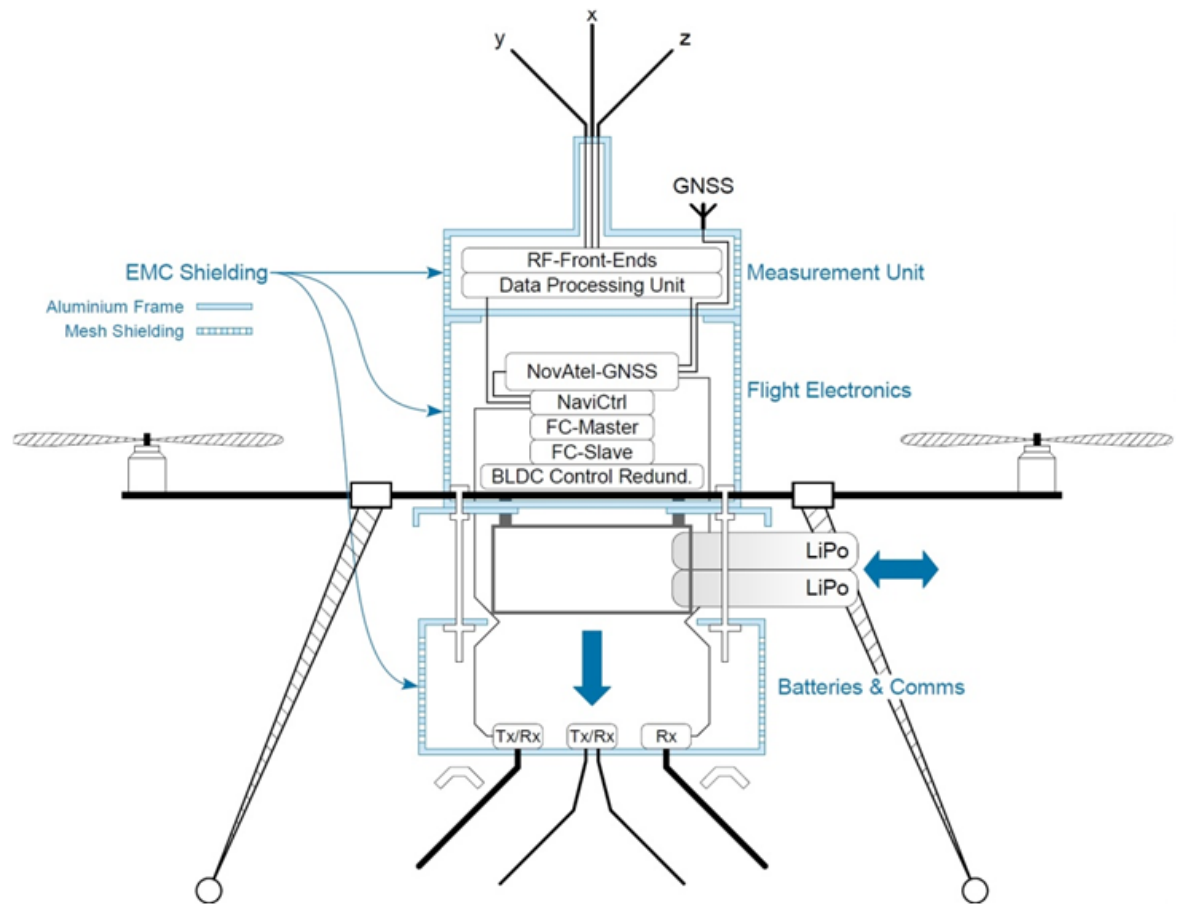

Figure 3. Mechanical setup of the UAS with the battery tray opened.

the yaw angle, but will also interfere with the fields to be measured, especially at frequencies up to a few hundreds of MHz. On the other hand, we plan to fly in electromagnetic harsh environments. Therefore, shielding against high power RF external sources is necessary to protect the UAS itself. Of course, shielding against external RF radiation will also reduce the emissions from the UAS itself. Since payload is always limited on flying platforms, the shielding has to be of light weight. To reach the defined safety limits, all electronic instrumentation need to be encapsulated by the shielding, in- cluding motor controllers, flight controller, navigation controller, data sampling and storage unit, etc. To prevent overheating of the electronics inside, a mesh shielding has been designed that allows for some air flow through it. Another advantage of the mesh shielding is that the internal barometric pressure sensor of the UAS contributing to the height information relative to the ground level is not affected.

As the battery is covered by the shielding, the design has to allow a quick exchange of it. A low-weight aluminum frame with top and bottom plates and with mesh inserts forms the 


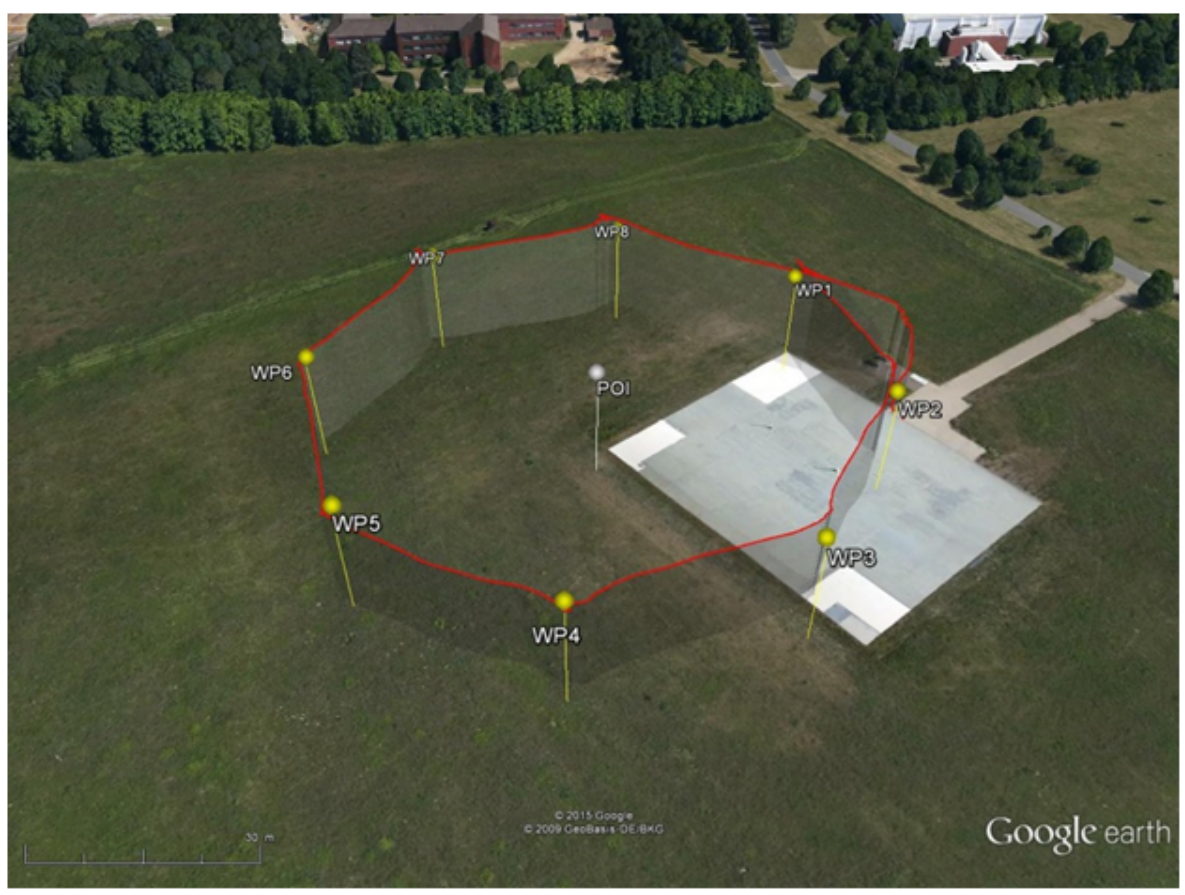

Figure 4. Flight track with waypoints (WP) and point of interest (POI).

outer body of the shielding that takes all of these conditions into account. The top plate also serves as mounting pad for the different sensing heads and the GPS antenna. The inner part of the bottom plate can be unlocked and released, thereby giving access to the battery compartment. The outer ring of the lower plate serves as support for the antenna of the remote-control receiver, the downlink for the flight status to be received by a smartphone application, and the uplink for the RTK correction signal. All input and output signal paths are filtered using narrowband bandpass filters.

For simplicity reasons, the sensing head is typically mounted on top of the UAS, which moves the center of gravity of the UAS. Test flights with simple ballast instead of the electronics have shown, that the UAS can handle its payload even in windy conditions. The octocopter setup seems to be more stable compared to UAS configured with a smaller number of motors/propellers, especially in case of motor/controller failure. For some applications, e.g. when the measurement signal directly comes from "down under" the sensor is mounted underneath the UAS, using an updated calibration factor.

\subsection{Precision navigation using RTK}

One of the advantages of UAS is their ability to approach predefined waypoints (WP) (cp. Fig. 4), a locus in space (WGS84 format and height above ground - not absolute height), and to trigger some action when the WP has been reached. In our case, these WP are defined using the software MK-tool provided by HiSystems GmbH (Fig. 2). The tool uses georeferenced satellite images as maps. Once defined, the WP data are transmitted to the UAS with all necessary parameters such as horizontal and vertical (ascending/descending) speed, waypoint radius (WPR), dwell time at the waypoint, steering of the UAS towards a fixed cardinal direction or towards one or a set of predefined points of interest (POI). After the automatic start procedure, the microcopter floats to the first WP. The predefined WPR between 1 and $10 \mathrm{~m}$ determines the region in space around a WP, where the navigation controller assumes that the given waypoint has been reached. The manufacturer recommends a value of around $10 \mathrm{~m}$ for this parameter. Thus, this point reached by the microcopter may be apart from the desired point in space by a few meters, depending on the wind conditions, the actual payload and some parameters of the flight controller itself, and the actual satellite constellation. The typical accuracy of the GPS and GLONASS signals without differential GNSS improvement (d- GNSS) or real-time kinematic (RTK) will only enable accuracy of a few $\mathrm{m}$ in lateral position, but also for the altitude (Morgan-Owen and Johnston, 1995; Shao and Sui, 2015). We have tested the relative accuracy by repeated flight tests using the same flight pattern (cp. Fig. 5 flypath 1 and 2). The weather conditions were almost perfect - i.e. it was a sunny and not windy day (2-3 Bft). Flight tests were performed on our open area test site (OATS), which is a $50 \mathrm{~m}$ by $60 \mathrm{~m}$ size metal reflector on the ground with at least $150 \mathrm{~m}$ clearance around it. Thus, a maximum number of satellites can be received. For simplicity, we have chosen a circular track with $100 \mathrm{~m}$ diameter in $20 \mathrm{~m}$ height. The WPR was set 


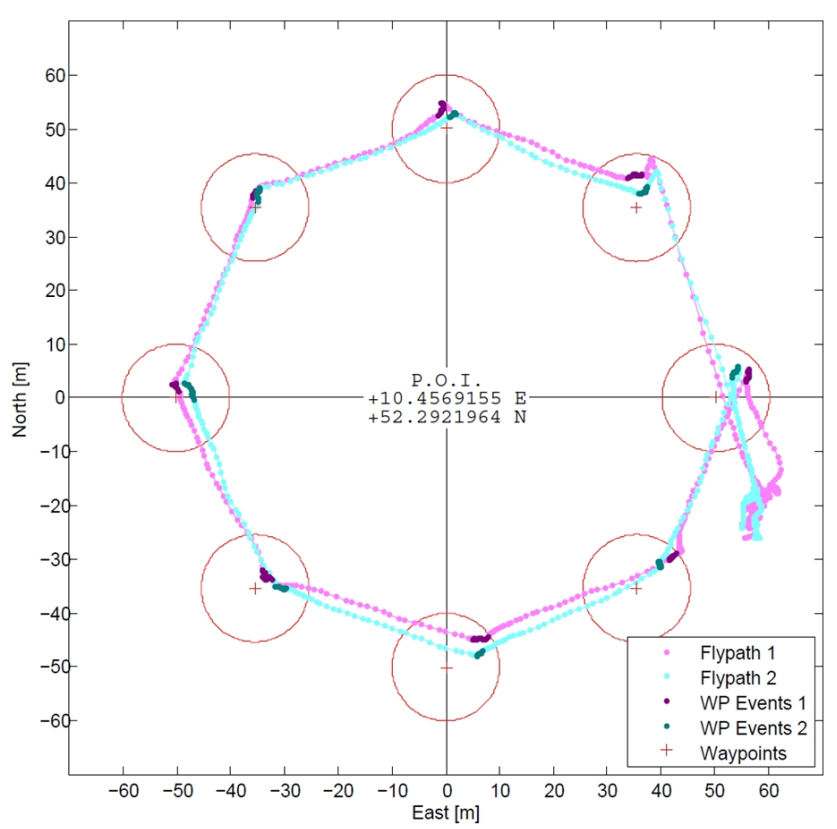

Figure 5. Repeated flight pattern using the same flight track with standard single frequency GPS receiver u-blox LEA 6S.

to $10 \mathrm{~m}$ (cp. the red circles in Fig. 5). When the UAS realized that the WP has been reached, it hovered there for $10 \mathrm{~s}$ and then continued its flight to the next WP. The time needed to catch the WP was almost negligible. Next, we have decreased the WP to $3 \mathrm{~m}$. It took the UAS quite a while (about $15 \mathrm{~s})$ to recognise the actual position being located inside the waypoint radius though it did not change its position. Further reduction of the WP radius caused the octocopter to stay at its position trying to reach the first WP. Especially under windy conditions and with high payload this localisation was not acceptable.

In order to be able to reduce the waypoint radius without sacrificing the time to reach the desired WP, the single frequency GPS receiver u-blox LEA 6S was removed and replaced by a OEM615 (NovAtel, 2013), which is a state-ofthe-art dual frequency airborne receiver. Its update rate for the actual position is improved to $20 \mathrm{~Hz}$ compared to $5 \mathrm{~Hz}$ before. Some additional hardware had to be designed in order to integrate the new receiver with the existing electronics (Fig. 6). Because u-blox and NovAtel use different protocols for transmitting the actual position information, the software of the navigation controller was also modified to accommodate a new protocol parser. Besides the signals from GPS and GLONASS satellites the NovAtel receiver uses additional differential correction data in RTCA or RTCM format from a NovAtel ground station (receiver model OEM628; NovAtel, 2013). The latter acquires samples from the GNSS satellite information for a long observation time, e.g. $24 \mathrm{~h}$ or even more. This significantly reduces the uncertainty of the position of the ground station receiver, until it has reached a

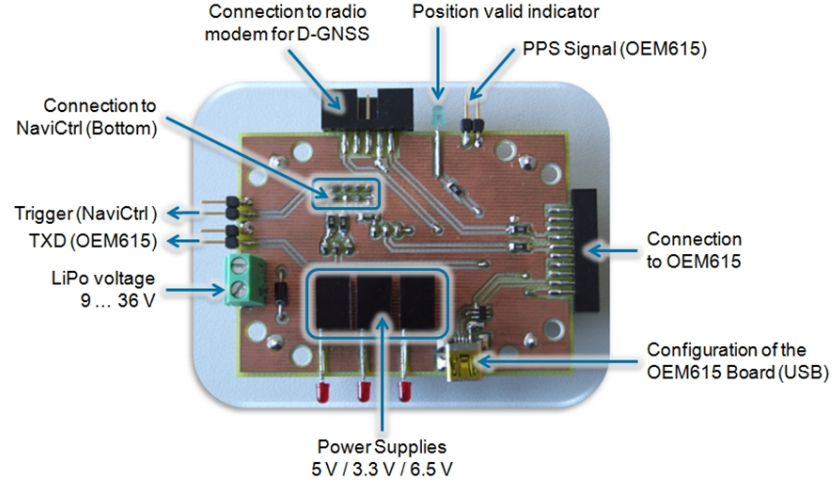

Figure 6. Connector board between NovAtel receiver, radio modem, and UAS.

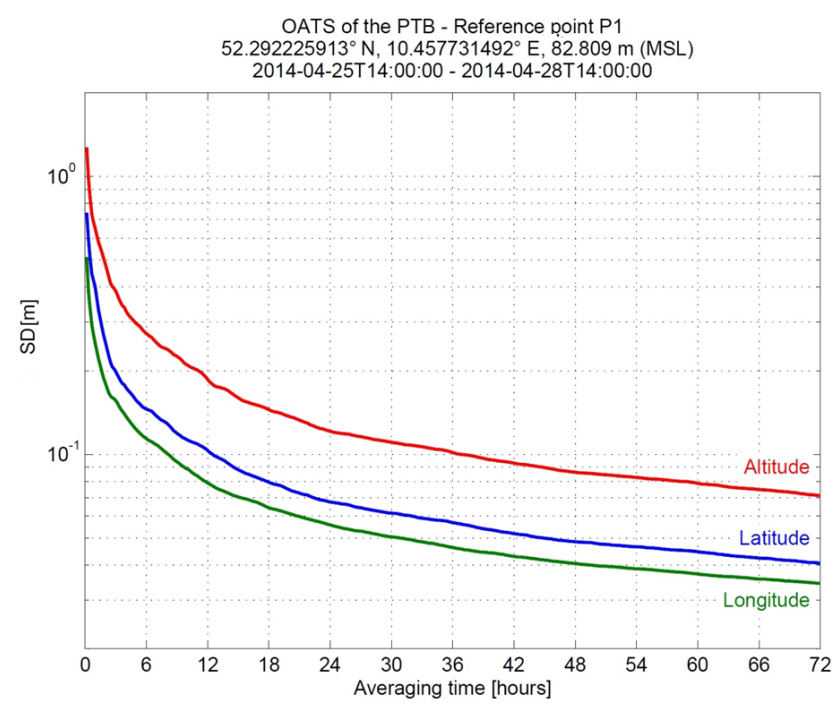

Figure 7. Standard deviation of the NovAtel ground station receiver position.

lateral uncertainty of position of a few $\mathrm{cm}$ and a height accuracy of better than $10 \mathrm{~cm}$ (Fig. 7). From this known position and the actual GNSS information the ground station receiver calculates the offset and transmits the error correction using a radio link with an update rate of $1 \mathrm{~Hz}$. The onboard receiver of the UAS takes this correction signal together with the actual GNSS data to calculate the updated position as input for the navigation controller of the UAS.

A test of the NovAtel ground station and a stationary operated UAS showed the drastic improvement in localisation close to the theoretical limit. Further tests with payload and under windy conditions will reveal the practical limit of the advanced positioning.

During its operation the UAS transmits the actual position of the UAS including the height, the time stamp, and the validity of the measurement data using another radio link. This flight log data can be displayed in real-time on an Androidbased smartphone, for which an application has been written. 


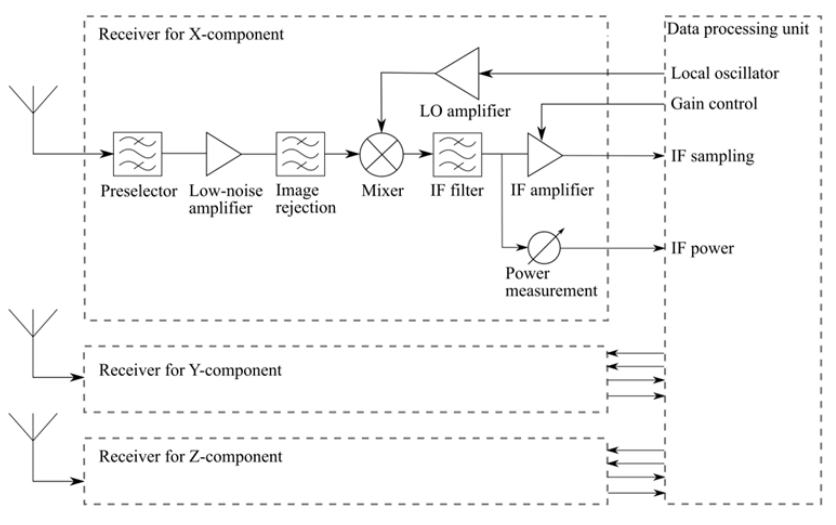

Figure 8. Block diagram of the RF frontend.

\section{The instrumentation and data processing unit}

\subsection{RF front-ends and antennas}

As already mentioned, the applications for the UAS-based platform are quite versatile including the measurement of radio frequency (RF) signals. For every application different $\mathrm{RF}$ frontends will be developed, which can be connected to the data processing unit using the same interface (Fig. 8). Until now, a superheterodyne receiver design is used (Pozar, 2005). All receivers provide a $50 \Omega$ input connector for the antenna. The RF signal is amplified, filtered and downconverted to an intermediate frequency (IF) of $70 \mathrm{MHz}$ using a local oscillator signal from the data processing unit. The IF signal is then filtered by a Surface-Acoustic-Wave (SAW) filter, to suppress undesired mixing products and adjacent RFchannels. The IF-signal is amplified and can be measured at a $50 \Omega$ output port. The IF signal is sampled and stored by the data processing unit using a fast analog-to-digital converter (ADC) and a solid-state hard disc as mass storage device. To obtain a higher dynamic range a variable gain amplifier (VGA) is used as an IF amplifier (DVGA2-33+ from MiniCircuits, http://www.minicircuits.com). The gain can be controlled by the data processing unit via a serial interface. To determine the correct gain, the IF power is measured after the IF filter using a logarithmic amplifier with demodulation (AD8307 from Analog Devices, http://www.analog.com). The device delivers a DC voltage that is proportional to the logarithm of the input voltage level, which then can be measured by the data processing unit using an ADC. Until now, highly integrated amplifiers are used for the receiver providing high gain, low noise figure, and high bandwidth (Fig. 9). Additionally, the receivers design can easily be adapted for new applications. Only a few frequency dependent components like the input and IF filters need to be replaced. However, the power consumption of approx. $300 \mathrm{~mW}$ for each receiver is relatively high.

As an example the electrical field strength in free space can be measured by calibrating the whole system, including

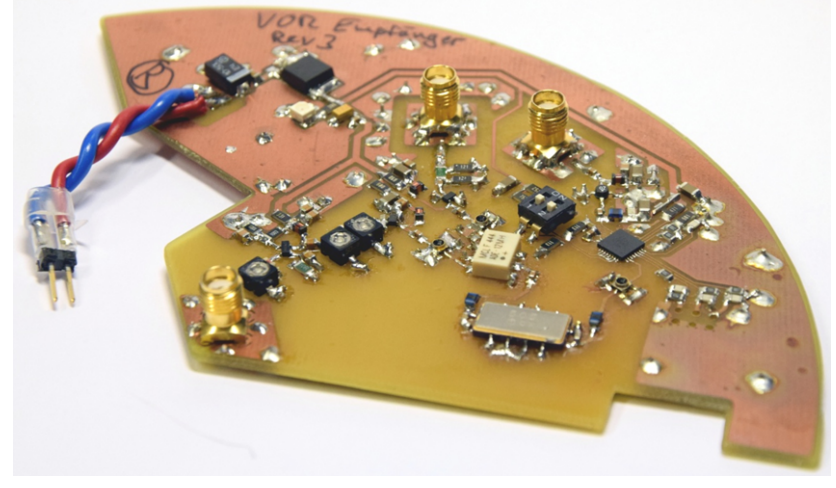

Figure 9. Photograph of one receiver channel board.

the antenna, the RF frontend, and the data processing unit, in a known electrical field.

The data processing unit is capable to sample up to 3 received signals simultaneously. Therefore, the electrical field vector can be calculated measuring the field strength of 3 probes where each probe is sensitive for one field component in an orthogonal system. Of course, scalar or 2-dimensional measurements are also possible. A variety of linear antennas has been developed for different frequencies and applications. Measurements have been performed with passive dipoles, active dipoles, passive bowtie antennas and passive monopoles. Because of the limited space on the UAS, electric small antennas are preferred to detect electromagnetic waves up to $1 \mathrm{GHz}$. For simplicity, the radiation pattern of each antenna should be as close as possible to an ideal, electrical short, linear antenna (Balanis, 2005). Since the radiation pattern is not ideal, a model for real antennas has been developed. The signal of the real antenna is modelled as a superposition of the signals of an ideal isotropic and an ideal linear antenna. The parameters for this model are again determined through calibration in a known electrical field.

\subsection{Data acquisition and processing}

It is the task of the data acquisition board to control the receiver frontend and to pre-process and save the RF data on a suitable storage medium. Figure 10 gives an overview of the components.

An "Arria V" FPGA is the central design unit that controls all components and employs an embedded 32bit processor (Altera, www.altera.com). A coherent clock distribution fed by a stable temperature-controlled crystal oscillator (TCXO) drives all frequency-dependent components as local oscillators (LO) and A/D converters. Three receivers can be connected that allow to measure the field polarisation with an orthogonal antenna-triple. The 16 bit-wide A/D converters have sample rates up to $160 \mathrm{MHz}$. That ensures to sample wideband signals of radar applications within WERAN.

LO frequencies are directly generated on the board using integrated PLL/VCO chips. As stated above, their signals 


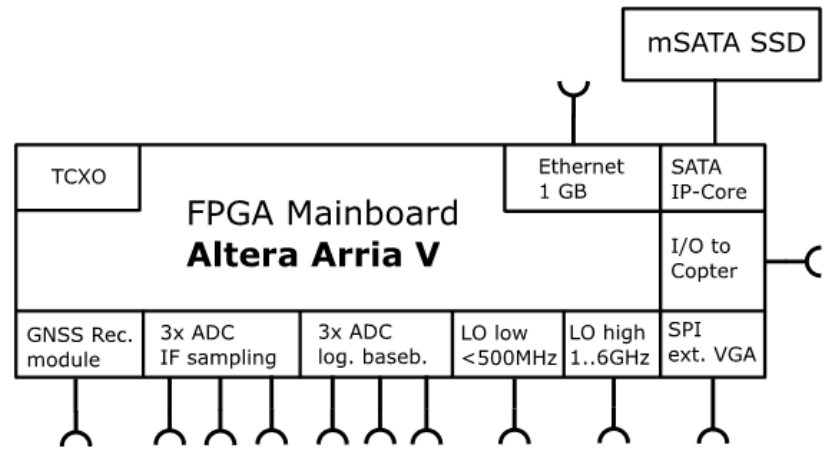

Figure 10. Block schematics of data acquisition and signal processing board.

are fully coherent with $\mathrm{AD}$ sampling. That simplifies the RF frontends which hence do not need own LOs. Actually, the $\mathrm{RF}$ can be provided with two LO frequencies. This enables RF concepts with two intermediate frequencies (IF) which is useful at carrier frequencies of several GHz. The control board of each RF frontend also includes an automatic gain control (AGC) to adapt the RF signal strength to the ADC input. Variable gain amplifiers (VGA) are connected via a serial peripheral interface (SPI).

RF data acquisition is performed by direct IF sampling, i.e. a band pass signal (but not a baseband signal) is obtained. Any necessary signal pre-processing is carried out within the FPGA. However, it is the concept of the WERAN data processing that a nearly unaltered signal is stored on the octocopter platform to be analyzed by subsequent post processing. A high-bandwidth data recording is realized by implementing a SATA-IP (Serial ATA disk) core in the FPGA. It operates at SATA 3-speed (6 GBps) and allows the direct connection of a micro SATA solid-state disk (SSD). A LAN interface allows for the download of the measurement data after landing of the octocopter without removing the SSD from the UAS. The processor interfaces with the main octocopter controller via an I/O interface. Optionally, it can use its on-board GNSS module to obtain time and position information.

\subsection{Undersampling technique}

A common misconception of the Nyquist criteria is that the sampling frequency must be twice the highest frequency present in the signal $s(n)$. In fact, the sampling frequency needs to be only twice the signal bandwidth to prevent aliasing:

$f_{\mathrm{S}}>2 \Delta f$

Undersampling is corresponding with the use of a sampling frequency which is less than the highest frequency present in the signal. In Fig. 11, from (Zumbahlen, 2008) this principle is illustrated in frequency domain. Signals below
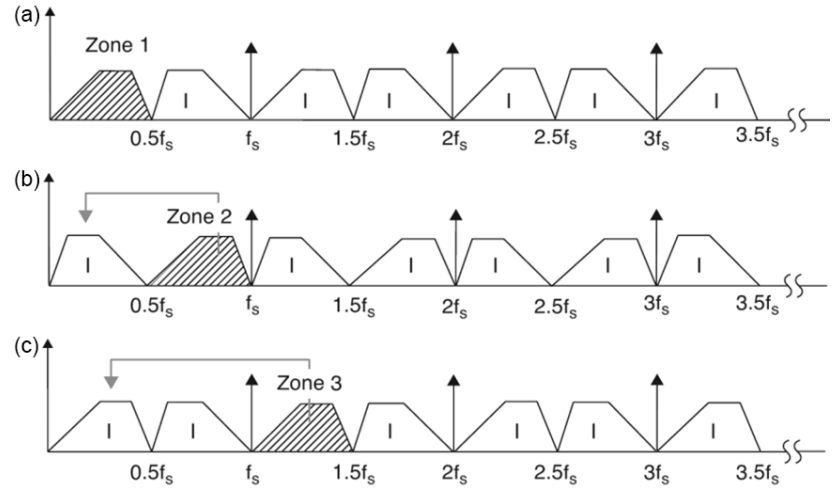

Figure 11. Nyquist zones and frequency translation (Zumbahlen, 2008).

$0.5 f_{\mathrm{S}}$ are located in the so-called first Nyquist Zone (NZ), see panel a. Sampling these signals preserves their original carrier frequency. The number of NZ increments by every $0.5 f_{\mathrm{S}}$. Frequencies higher than half the sampling rate are folded back into the 1st NZ. Hence, a signal located in any higher NZ will give an image in the first NZ (panel b, c). No signal information is lost except for the value of the original carrier frequency $f_{\mathrm{C}}$. An additional frequency reversal occurs, if signals are located in even Nyquist zones (panel b).

If equation

$f_{\mathrm{S}}=\frac{4 f_{\mathrm{C}}}{2 \mathrm{NZ}-1}$

applies, then the image of $f_{\mathrm{C}}$ is safely placed in the center of the first $\mathrm{NZ}\left(=0.25 f_{\mathrm{S}}\right.$; Zumbahlen, 2008).

Then the primary function of an anti-aliasing filter is to ensure that the band of sampled signals must not overlap any multiple of $f_{S} / 2$, i.e. it is limited to a unique Nyquist zone. In the WERAN RF frontend designs a steep band pass filter is placed in the IF section. The VOR RF frontend offers a $70 \mathrm{MHz}$ IF. After further amplification on the digitizer board, this signal is then initially sampled at $105 \mathrm{MHz}$. This converts the IF into the second NZ, resulting in an image in first NZ at $35 \mathrm{MHz}$. A chain of FIR (Finite Impulse Response) decimation band pass filters inside the FPGA then reduces the sampling rate dramatically, but finally it remains well above twice the channel bandwidth of $25 \mathrm{kHz}$ when stored on the SSD.

\section{First measurements}

Some reception tests were carried out at the Doppler-VOR "Hehlingen" (HLZ), Germany, using a static receiver antenna on PTB premises at a distance of about $23 \mathrm{~km}$ to the DVOR (measurement antenna located at the Willy-Wien tower about $40 \mathrm{~m}$ above ground level). Applying a Discrete Fourier Transformation (DFT) to the discrete in time and am- 


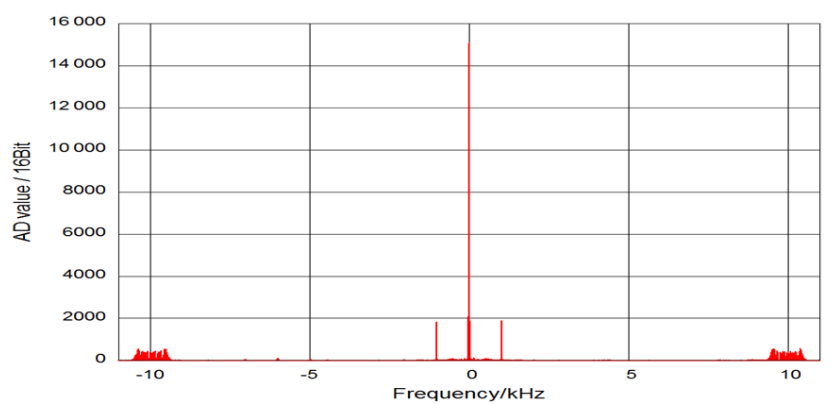

Figure 12. Bandpass signal of received DVOR "HLZ" at 117.3 MHz; static measurement on PTB premises at a distance of about $23 \mathrm{~km}$ to the DVOR (measurement antenna located at the Willy-Wien tower about $40 \mathrm{~m}$ above ground level).

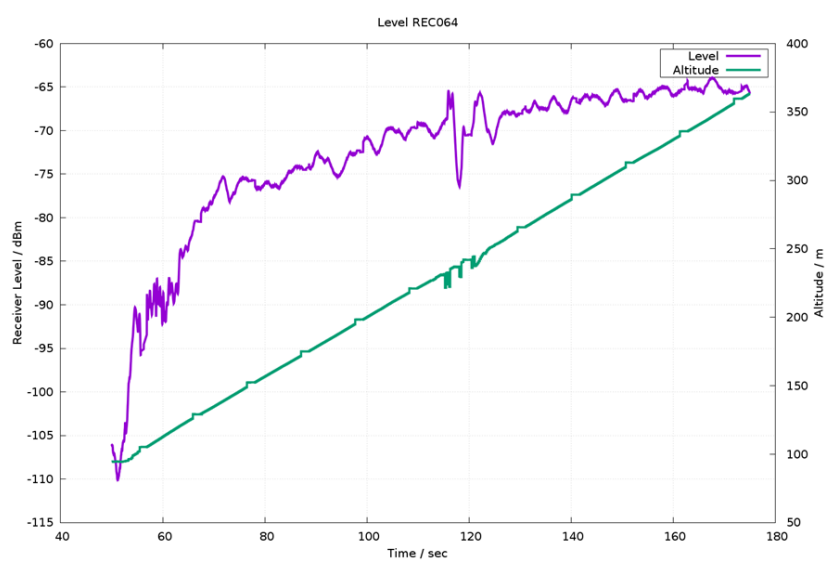

Figure 13. Measured receiver level of DVOR Wolfsburg-Hehlingen (Germany) as a function of altitude.

plitude band pass samples gives a spectrum normalized to the carrier frequency (Fig. 12).

In the first measurement campaign the DVOR "HLZ" in Wolfsburg-Hehlingen, Germany, was investigated. As an example for the data obtained, Fig. 13 shows a cut-out of the measured receiver level as a function of altitude. After automatic start, the octocopter was programmed to constantly increase its altitude up to $500 \mathrm{~m}$ at $2.5 \mathrm{~m} \mathrm{~s}^{-1}$ speed. The platform maintained that position for $30 \mathrm{~s}$, followed by the automatic decay down to $30 \mathrm{~m}$ altitude, again at constant $2.5 \mathrm{~m} \mathrm{~s}^{-1}$ speed. Landing is typically performed by manual maneuvers. Latitude and longitude were kept the same during the flight. The starting point was located about $5 \mathrm{~km}$ south of the DVOR in the open landscape, without any buildings, wind turbines etc. in the line-of-sight.

\section{Conclusions}

Design and concept of a new versatile and flying measurement platform used to perform precision measurements of electromagnetic signals in space are described. This un- manned aerial system (UAS) is based on a commercially available octocopter. Its navigation and localisation capabilities were significantly improved by a state-of-the-art realtime kinematic receiver and a ground station transmitter, which delivers the correction signal for the actual GNSS signal. The on-board receiver utilises the actual GNSS signal and the correction to determine an improved position in space, which is fed into the navigation and flight controller of the UAS. Automatic starts, flights along predefined tracks using waypoints, and automatic landing makes the UAS easy to operate. The alignment of the "front" direction of the UAS with respect to a point of interest allows the measurement of radiation pattern in space. With the availability of this UAS remote areas now become easily accessible, especially if an elongated observation time for measurements is needed. Flight costs are at low level. Flight time with one set of batteries is in the order of $30 \mathrm{~min}$ depending on the payload and the wind conditions. To fly in electromagnetic harsh environments, a proper shielding of the electronics was considered and the final tests are underway. Heating up inside the shielding was avoided by using a mesh cover. To ensure its proper operation, the main parameters of the UAS are transmitted and may be displayed on an Android-based smartphone.

A set of antennas and receivers is currently developed for each frequency band of interest, starting at the VOR frequencies of around $110 \mathrm{MHz}$. So far, the highest measurement frequency is $5.6 \mathrm{GHz}$ (weather radars). The sensing heads are interchangeable using a lock to make the mounting position consistent and repeatable, thereby maintaining the validity of the calibration factors. Antenna calibration must be performed on the whole set consisting of the UAS and the sensing head. Straight forward superheterodyne receivers in the $\mathrm{RF}$ frontends feed the measurement signals into the FPGAbased sampling board. RF data acquisition is done by direct IF sampling, i.e. a band pass signal (but not a baseband signal) is obtained. As the raw data are stored on a solid-state disk, all options are available for post-processing.

First measurement results indicate the expected performance and document the proof-of-concept.

Acknowledgements. The authors thank their partners within the WERAN project: Mr. Heyno Garbe and his team from the Leibniz University of Hanover (LUH), steep $\mathrm{GmbH}$, as well as Deutsche Flugsicherung, Deutscher Wetterdienst, and Bundesaufsichtsamt für Flugsicherung for continuing support and discussion.

Edited by: T. Probst

Reviewed by: two anonymous referees 


\section{References}

Balanis, C. A.: Small Dipole, in: Antenna Theory, 3rd Edn., Hoboken NJ, John Wiley \& Sons Inc., 2005.

Mihalachi, M., Schrader, T., Rohde, J., and Kleine-Ostmann, T.: UAV-Based Measurement Platform for Precision Electromagnetic Field Measurements, 2015 IEEE International Symposium on Electromagnetic Compatibility (EMC), WS14 Unmanned Aircraft Systems - EMC and Applications, Dresden, Germany, 16-22 August, 2015.

Morgan-Owen, G. J. and Johnston, G. T.: Differential GPS positioning, Electron. Commun. Eng., 7, 11-21, 1995.

NovAtel: Positioning Modes of Operation, Application Note, Rev. 1, http://www.novatel.com/assets/Documents/Bulletins/apn051. pdf, 24 October 2013.
Pozar, D. M.: Radio Receiver Architectures, in: Microwave Engineering, 3rd Edn., John Wiley \& Sons Inc., 2005.

Schrader, T., Bredemeyer, J., Stupperich, C., and Garbe, H.: WERAN - Interaction of Wind Turbines with Terrestrial Navigation/Radar Systems, 2015 IEEE International Symposium on Electromagnetic Compatibility (EMC), WS14 Unmanned Aircraft Systems - EMC and Applications, Dresden, Germany, 1622 August, 2015.

Shao, M. and Sui, X.: Study on Differential GPS Positioning Methods, 2015 International Conference on Computer Science and Mechanical Automation (CSMA), 23-25 October 2015, 223 225, 2015.

Zumbahlen, H. (Ed.): Linear Circuit Design Handbook, Analog Devices, 2008. 